\title{
Low expression of miR-532-3p contributes to cerebral ischemia/reperfusion oxidative stress injury by directly targeting NOX2
}

\author{
LI MAO ${ }^{1,2^{*}}$, MEI-LING ZUO ${ }^{1,3^{*}}$, AI-PING WANG ${ }^{4}$, YING TIAN $^{4}$, LI-CHEN DONG $^{1}$, \\ TAO-MING LI ${ }^{1}$, DA-BIN KUANG ${ }^{1}$, GUI-LIN SONG ${ }^{1,3}$ and ZHONG-BAO YANG ${ }^{1,3}$
}

\author{
${ }^{1}$ Office of Good Clinical Practice, The Affiliated Changsha Hospital of Hunan, Normal University, Changsha, \\ Hunan 410006; ${ }^{2}$ Department of Basic Medicine, Changsha Health Vocational College, Changsha, Hunan 410100; \\ ${ }^{3}$ Institute of Emergency and Critical Care Medicine of Changsha, Changsha, Hunan 410006; ${ }^{4}$ Institute of Clinical \\ Research, Affiliated Nanhua Hospital, University of South China, Hengyang, Hunan 421001, P.R. China
}

Received November 26, 2019; Accepted May 21, 2020

DOI: $10.3892 / \mathrm{mmr} .2020 .11325$

\begin{abstract}
NADPH oxidase 2 (NOX2) is a major subtype of NOX and is responsible for the generation of reactive oxygen species (ROS) in brain tissues. MicroRNAs (miRNAs/miRs) are important epigenetic regulators of NOX2. The present study aimed to identify the role of NOX2 miRNA-targets in ischemic stroke (IS). A rat cerebral ischemia/reperfusion (CI/R) injury model and a SH-SY5Y cell hypoxia/reoxygenation $(\mathrm{H} / \mathrm{R})$ model were used to simulate IS. Gene expression levels, ROS production and apoptosis in tissue or cells were determined, and bioinformatic analysis was conducted for target prediction of miRNA. In vitro experiments, including function-gain and luciferase activity assays, were also performed to assess the roles of miRNAs. The results indicated that NOX2 was significantly increased in brain tissues subjected to $\mathrm{I} / \mathrm{R}$ and in SH-SY5Y cells subjected to $H / R$, while the expression of miR-532-3p (putative target of NOX2) was significantly decreased in brain tissues and plasma. Overexpression of miR-532-3p significantly suppressed NOX2 expression and ROS generation in SH-SY5Y cells subjected to H/R, as well as reduced the relative luciferase activity of cells transfected with a reporter gene plasmid. Collectively, these data indicated that miR-532-3p may be a target of NOX2 and a biomarker for $\mathrm{CI} / \mathrm{R}$ injury. Thus, the present study may provide a novel target for drug development and IS therapy.
\end{abstract}

Correspondence to: Dr Zhong-Bao Yang, Office of Good Clinical Practice, The Affiliated Changsha Hospital of Hunan, Normal University, 70 Lu-Shan Road, Changsha, Hunan 410006, P.R. China E-mail: yzb55@yahoo.com

*Contributed equally

Key words: cerebral ischemia/reperfusion injury, oxidative stress, NADPH oxidase 2, microRNA-532-3p, reactive oxygen species

\section{Introduction}

Ischemic stroke (IS) is an age-related central nervous system disease that occurs worldwide, and is associated with high mortality and morbidity rates, as well as large economic and psychological burdens to patients and their families (1). When the blood supply of the brain vessels is interrupted, the affected brain tissues that lack an adequate oxygen and glucose supply suffer severe damage and death, which leads to IS (2). Currently, the most common treatment strategy for IS is recovering the blood supply of the brain using methods such as thrombolysis (1). However, reperfusion of blood to the brain results in severe injury of the affected neuron cells, which is termed cerebral ischemia/reperfusion (CI/R) injury (3). Numerous factors contribute to the pathogenesis of $\mathrm{CI} / \mathrm{R}$ injury, such as oxidative stress, excitotoxicity and edema $(2,4)$. Moreover, oxidative stress is reported to serve pivotal roles in CI/R injury (4); however, its exact mechanism is yet to be elucidated. Therefore, identifying the mechanism of oxidative stress in CI/R injury has become an important topic of research in IS therapy and drug development.

NADPH oxidase (NOX) is a critical enzyme for the production of reactive oxygen species (ROS) in several cell types, such as neutrophils, white blood cells and neurons (5). Furthermore, NOX mediates the electron transport between NADPH and oxygen and generates superoxide anion (a reactive free-radical), which is the primary cause of oxidative stress injury (6). Previous studies have reported that seven isoforms of NOX exist in mammalian cells, including NOX1-NOX5, Dual Oxidase (DUOX)1 and DUOX2 $(5,6)$. In addition, our previous study revealed that NOX4, and particularly NOX2, were the primary subtypes responsible for ROS generation in $\mathrm{CI} / \mathrm{R}$ injury (6). When subjected to $\mathrm{I} / \mathrm{R}$ injury, the expression of NOX2 in affected brain tissues is significantly upregulated and is followed by ROS overproduction (6). In addition, middle cerebral artery occlusion (MCAO) rats administrated with a NOX2 inhibitor, such as apocynin, exhibit reduced cerebral infarction and improved neurological function (7). These findings suggest that NOX2 is a promising target for the treatment 
of CI/R injury. Therefore, elucidating the regulatory mechanism of NOX2 expression is important for the understanding of $\mathrm{CI} / \mathrm{R}$ injury, and thus requires further investigation.

MicroRNAs (miRNAs/miRs) are a class of epigenetic regulators, with a length of 21-24 nucleotides, which serve a role in gene expression and are involved in the pathogenesis of numerous diseases, including IS (8). The expression levels of miRNAs have been identified to be altered in the brain tissues of rats when subjected to MCAO, such as miR-107 (9-11). Although previous studies have shown that miR-126a-3p and miR-138-5p are targets of NOX2 (12), further research is required to examine the target miRNAs of NOX2 in CI/R injury. miR-532 is a multifunctional molecule and has important roles in multiple biological processes. For example, miR-532 mitigates cardiomyocyte apoptosis caused by hypoxia by targeting the pyruvate dehydrogenase complex associated with energy homeostasis in the heart $(13,14)$. In addition, miR-532 is an important molecule in the pathogenesis of several central neuronal system diseases, including IS $(15,16)$. However, to the best of our knowledge, whether there is an interaction between miR-532 and NOX2 has not yet been investigated. Therefore, examining the roles of miR-532 in the regulation of NOX2 is important for the understanding and treatment of IS.

The present study was conducted to assess whether miR-532-3P is a target miRNA of NOX2 and to identify the role of miR-532-3p in CI/R oxidative stress injury. To the best of our knowledge, the present study is the first to investigate the role of the miR-532-3p/NOX2 axis in CI/R injury. Therefore, the results may provide a novel target for drug development and IS therapy.

\section{Materials and methods}

Animal experiments. A total of 16 male Sprague-Dawley rats (weight, 240-260 g; age, 8 weeks) were purchased from the Hunan SJA Laboratory Animal Co., Ltd., and a rat CI/R injury model was established following a previously described surgical method (11). Rats were subjected to a $2 \mathrm{~h}$ occlusion of the left internal carotid artery and a $24 \mathrm{~h}$ reperfusion. During the whole experimental process, the animals were housed under standard conditions $\left(25^{\circ} \mathrm{C} ; 65 \%\right.$ humidity; an alternative of $12 \mathrm{~h} \mathrm{light} / 12 \mathrm{~h}$ dark cycle) with free access to food and water. In total, $3 \%$ pentobarbital sodium ( $40 \mathrm{mg} / \mathrm{kg}$ weight) was used for light anesthesia. During the surgical course, the body temperature of the rat was maintained at $\sim 37^{\circ} \mathrm{C}$. The duration of the experiments was $\sim 26 \mathrm{~h}$ (including $2 \mathrm{~h}$ ischemia and $24 \mathrm{~h}$ reperfusion). During ischemia, vital signs of rats were monitored, such as body temperature and respiration. During reperfusion, vital signs of rats were monitored every $6 \mathrm{~h}$. Animal experiments were conducted following the Guide for the Care and Use of Laboratory Animals published by the National Institute of Health (17), and were approved by the Animal Ethics Committee of Hunan Normal University. There were eight rats used in each group (I/R and sham). The sham group was subjected to a skin incision without occlusion of the left internal carotid artery.

At the end of the $24 \mathrm{~h}$ reperfusion, all rats (16 rats) were sacrificed by decapitation after loss of consciousness following anesthesia via intraperitoneal injection of $3 \%$ pentobarbital sodium (40 mg/ $\mathrm{kg}$ weight). The neurological function was evaluated following a $24 \mathrm{~h}$ reperfusion, and subsequently the brain tissue was collected for 2,3,5-tripenyltetrazolium chloride (TTC) staining or other assays, including the detect of protein and mRNA expression levels.

Cell culture. The SH-SY5Y cell line (neuroblastoma) was provided by the Committee on Type Culture Collection of Chinese Academy of Sciences of Shanghai. Short-tandem repeat profiling of SH-SY5Y cell line was performed prior to experiments. A compound culture was used for cells culture, which consisted of DMEM (Thermo Fisher Scientific, Inc.), 10\% FBS and penicillin/streptomycin $(100 \mathrm{U} / \mathrm{ml})$. Cells were maintained in a cell incubator at standard conditions $\left(37^{\circ} \mathrm{C}, 5 \% \mathrm{CO}_{2}\right.$ and $95 \%$ air). Cells $\left(5 \times 10^{5}\right)$ were cultured with 12-well plates for miRNA functional assays and the luciferase reporter gene experiment.

Cell model of hypoxia/reoxygenation $(H / R)$. An in vitro SH-SY5Y cell H/R model was established to mimic IS or CI/R injury. When cells reached $70 \%$ confluence, DMEM culture was removed and replaced with Dulbecco's phosphate-buffered saline (DPBS; Sigma-Aldrich; Merck KGaA). Then, cells were maintained at $37^{\circ} \mathrm{C}$ in a hypoxic condition $(95 \%$ $\mathrm{N}_{2}$ and $5 \% \mathrm{CO}_{2}$ ) for $5 \mathrm{~h}$. After the $5 \mathrm{~h}$ hypoxia process, DPBS was removed and the compound DMEM culture was added and cells were maintained under standard conditions $\left(37^{\circ} \mathrm{C}\right.$, $5 \% \mathrm{CO}_{2}$ and $95 \%$ air) for $20 \mathrm{~h}$ reoxygenation.

Assessment of brain injury. To evaluate the degree of brain injury, a 5-point rating scale was used for neurological function assessment. According to the scale, 0 indicated the rat had no deficit, 1 indicates the rat was unable to spread the left forepaw, 2 indicates the rat's grasp strength of the left forepaw was reduced, 3 indicates the rat circling to the left on pulling of the tail and 4 indicates the rat is spontaneously circling (6).

Brain infarct was assessed using TTC staining (ischemic hemisphere and non-ischemic contralateral side appear while or red, respectively). Brain slices were prepared by coronally cutting brain tissues into sections with a thickness of $0.2-0.3 \mathrm{~cm}$. Then, the slices were immersed in $2 \%$ TTC and maintained in dark $\left(37^{\circ} \mathrm{C}\right)$ for $0.5 \mathrm{~h}$. Stained brain tissues were scanned and the infarct volume was calculated using ImageJ software (version 1.4; National Institutes of Health). The infarction volume $\left(\mathrm{cm}^{3}\right)$ of each slice was calculated using the following equation, which was described in our previous study (11): Infarction volume of each slice=infarct size $\left(\mathrm{cm}^{2}\right)$ of each slice $\mathrm{x}$ thickness.

Determination of NOX activity. To measure the total NOX enzyme activity, a colorimetric commercial kit (cat. no. GMS50095.1; Genmed Pharmaceutical Technology Co., Ltd.) was used according to the manufacturer's instructions. A mixture $(90 \mu \mathrm{l})$ of cell or tissues lysates (Cell lysis buffer for Western and IP; cat. no. P0013; Beyotime Institute of Biotechnology) and oxidized cytochrome c $(2 \mu \mathrm{l})$ was prepared and aliquoted into a quartz cuvette. After the mixture was reacted for $3 \mathrm{~min}$ at $30^{\circ} \mathrm{C}, 2 \mu \mathrm{lNADPH}$ was added to create a reaction solution. Then, the reaction solution was incubated at $30^{\circ} \mathrm{C}$ for $15 \mathrm{~min}$. Subsequently, NOX activity was determined by measuring the absorbance of the solution at $550 \mathrm{~nm}$ using a spectrophotometer. 
Determination of caspase-3 activity. To measure caspase-3 activity, a commercial kit (cat. no. C1116; Beyotime Institute of Biotechnology) was used according to the manufacturer's instructions. A mixture of cell or tissue lysate and caspase-3 substrate (Ac-DEVD-pNA; $10 \mu \mathrm{l}$ ) was prepared and incubated at $37^{\circ} \mathrm{C}$ for $60 \mathrm{~min}$. Then, caspase-3 enzymatic activity was determined by measuring the absorbance of the reaction solution at $405 \mathrm{~nm}$ using a multiscan spectrum (Thermo Fisher Scientific, Inc.). In total, one caspase-3 enzymatic activity unit refers to the quantity of enzyme required to catalyze $1.0 \mathrm{nM}$ substrate in $1 \mathrm{~h}$ at $37^{\circ} \mathrm{C}(6)$.

TUNEL/Hoechst double-labeling. A TUNEL assay kit (cat. no. C1086; Beyotime Institute of Biotechnology), and a Hoechst 33342 kit (cat. no. C1027; Beyotime Institute of Biotechnology) were used for the brain tissue cellular apoptosis assay. The assay process was conducted according to the method (TUNEL/Hoechst double-labeling) established by Whiteside et al (18). The sections of brain tissue (thickness, $5 \mu \mathrm{m})$ underwent the following steps: Fixed with formaldehyde $(4 \% \mathrm{w} / \mathrm{v})$ at $25^{\circ} \mathrm{C}$ for $10 \mathrm{~min}$, rinsed with PBS and post-fixed with formaldehyde and acetic acid at $4^{\circ} \mathrm{C}$ for $5 \mathrm{~min}$, washed with PBS, incubated with equilibration buffer and working strength deoxynucleotide transferase at $37^{\circ} \mathrm{C}$ for $1 \mathrm{~h}$, washed with PBS and incubated with Hoechst 33342 at $25^{\circ} \mathrm{C}$ for $5 \mathrm{~min}$. Slices were sealed using neutral balsam and examined using an epifluorescence microscope (Nikon Eclipse 80i) at x200 magnification in eight randomly selected fields of view. TUNEL-positive cells indicated apoptotic cells.

Hoechst staining. A commercial kit (Hoechst 33258; cat. no. C1017; Beyotime Institute of Biotechnology) was used to evaluate the apoptosis of SH-SY5Y cell. According to the manufacture's protocol, $\mathrm{SH}-\mathrm{SY} 5 \mathrm{Y}$ cells underwent the following steps: Fixed with formaldehyde $(4 \%)$ at $25^{\circ} \mathrm{C}$ for 10 min, rinsed with PBS, stained with Hoechst 33258 at $25^{\circ} \mathrm{C}$ for $5 \mathrm{~min}$ and imaged using a fluorescent microscope (magnification, x200). Cellular apoptosis was evaluated by calculating apoptotic percentage. Cellular apoptosis was calculated using the following formula: Number of apoptosis bodies/(number of apoptotic cells + number of cells).

Flow cytometry analysis. An Annexin V-FITC kit (cat. no. C1062M; Beyotime Institute of Biotechnology) was used to evaluate apoptosis of SH-SY5Y cells. According to the manufacturer's instruction, SH-SY5Y cells underwent the following steps: Incubated with FITC-conjugated Annexin V $(2.5 \%, \mathrm{v} / \mathrm{v})$ and propidium iodide (PI; 5\%, v/v) in the dark at $25^{\circ} \mathrm{C}$ for $20 \mathrm{~min}$, and then flow cytometry (BD FACSCalibur; BD Biosciences) was used to analyze cellular apoptosis. Cell apoptosis was analyzed using CellQuest Pro software (version 4.02; BD Biosciences). The rate of apoptosis was defined as the percentage of early and late apoptotic cells.

Bioinformatic prediction. TargetScan (www.targetscan. org/vert-72) and miRbase (www.mirbase.org) were used to predict the target miRNAs of NOX2.

Transfection with miR-532-3p mimics. To observe the function of miR-532-3p, an in vitro gain-of function experiment was performed using miR-532-3p mimics (5'-CCUCCCACACCC AAGGCUUGCA-3') and negative control miRNA (5'-UUC UCCGAACGUGUCACGUTT-3') (Guangzhou RiboBio Co., Ltd.). According to the manufacturer's protocols, a reaction mixture consisting of Lipofectamine ${ }^{\circledR} 2,000$ (Invitrogen; Thermo Fisher Scientific, Inc.) and miR-532-3p mimic was prepared and the final concentration of miR-532-3p mimic was $100 \mathrm{nmol}$. After $6 \mathrm{~h}$ of miR-532-3p mimic transfection, the mimic mixture was removed and replaced with standard media (DMEM supplement with 10\% FBS) for another $48 \mathrm{~h}$. Then, cells were collected for various analysis, such as western blotting and reverse transcription-quantitative PCR (RT-qPCR).

Luciferase reporter gene experiment. To determine the relationship between miR-532-3p and NOX2, a luciferase reporter gene plasmid (pGL6; Promega Corporation) containing a wild-type (WT) sequence (5'-GUGGGAGA-3') for miR-532-3p (defined as NOX2-WT) or a mutated (MU) sequence (5'-GUGAAAGA-3') for miR-532-3p (NOX2-MU) was constructed. The plasmids were purified by electrophoresis $(0.8 \%$ agarose gel supplemented with $0.5 \%$ ethidium bromide). Then, SH-SY5Y cells were co-transfected with $100 \mathrm{ng}$ plasmid (NOX2-WT or NOX2-MU) and miR-532-3p mimics or negative control miRNAs using Lipofectamine ${ }^{\circledR}$ 2000. At $6 \mathrm{~h}$ post-transfection, cells were incubated with DMEM supplemented with 10\% FBS for another $24 \mathrm{~h}$. Subsequently, cells were collected to assess enzyme activity. To evaluate the interaction between miR-532-3p and NOX2, a Dual Luciferase Reporter Gene Assay Kit (Beyotime Institute of Biotechnology) was used to measure the relative luciferase activity of SH-SY5Y cells. Firefly luciferase activities were normalized to Renilla luciferase activities.

DeterminationofROSlevels. To determinetheintracellular total ROS levels in SH-SY5Y cells, a Dichloro-dihydro-fluorescein diacetate (DCFH-DA) commercial kit (Beyotime Institute of Biotechnology) was used according to the manufacturer's instructions. SH-SY5Y cells or tissue homogenization were immersed in DCFH-DA $(10 \mu \mathrm{M})$ and maintained in dark for $20 \mathrm{~min}\left(37^{\circ} \mathrm{C}\right)$. The fluorescence signal of DCFH-DA at $502 \mathrm{~nm}$ was measured and the results are presented as arbitrary units.

Plasma. To obtain the plasma, blood was centrifuged at $3000 \mathrm{r} / \mathrm{min}$ for $10 \mathrm{~min}$ at $4^{\circ} \mathrm{C}$. The plasma was used for RNA extraction and RT-qPCR.

$R T-q P C R$. TRIzol $^{\circledR}$ reagent (Takara Biotechnology Co., Ltd.) was used for RNA extraction. Total RNA of each sample was obtained and purified prior to its concentration determination. A reverse transcription kit (cat. no. DRR037A; Takara Bio, Inc.) was used to obtain cDNA. The temperature protocol used for reverse transcription was as follows: $37^{\circ} \mathrm{C}$ for $5 \mathrm{~min}$ and $85^{\circ} \mathrm{C}$ for $5 \mathrm{sec}$. The RT PCR amplification reaction of NOX2 and miRNA was performed using ABI 7300 plus system (Applied Biosystems; Thermo Fisher Scientific, Inc.). SYBR Green (cat. no. SC200N/SC210N; Takara Bio, Inc.) was used for RT PCR. The following thermocycling conditions were used for qPCR: $95^{\circ} \mathrm{C}$ for $30 \mathrm{sec} ; 95^{\circ} \mathrm{C}$ for $5 \mathrm{sec}$; and $60^{\circ} \mathrm{C}$ for $31 \mathrm{sec}$ for 40 cycles. The PCR primers used 
were as follows: NOX2 forward, 5'-ACAAGGTTTATGACG ATGAGCC-3' and reverse, 5'-TTGAGCAACACGCACTGG AA-3'); $\beta$-actin forward, 5'-CCCATCTATGAGGGTTAC GC-3' and reverse, 5'-TTTAATGTCACGCACGATTTC-3'; miR-15a forward, 5'-UAGCAGCACAUAAUGGUUUGUG-3' and reverse, 5'-GTGCAGGGTCCGAGGT-3'; miR-16 forward, 5'-CGCGCTAGGAGCACGTAAATA-3' and reverse, 5'-GTG CAGGGTCCGAGGT-3'; miR-30a forward, 5'-GGTTGGCGC TCTGAGAGGTA-3' and reverse, 5'-GTGCAGGGGTCC GAGGT-3'; miR-125b forward, 5'-UCACCGGGUGUAAAU CAGCUU-3' and reverse, 5'-GTGCAGGGGTCCGAGGT-3'; miR-146a forward, 5'-GGGTGAGAACTGAATTCCA-3' and reverse, 5'-GTGCAGGGTCCGAGGT-3'; miR-153 forward, 5'-GGGATGGAGTCGAGGTGCGGCTAAT-3' and reverse, 5'-GTGCAGGGTCCGAGGT-3', miR-223 forward, 5'-UGU CAGUUUGUCAAAUACCCCA-3' and reverse, 5'-GTGCAG GGTCCGAGGT-3'; miR-532-3p forward, 5'-ACACTCCCC TCCCACACCCAAGG-3' and reverse, 5'-GTGCAGGGTCCG AGGT-3'; and U6 forward, 5'-CTCGCTTCGGCAGCACA-3' and reverse, 5'-AACGCTTCACGAATTTGCGT-3'. The 2- ${ }^{-\Delta C \mathrm{Cq}}$ method was used for data analysis and results were expressed as the ratio of NOX2 mRNA to $\beta$-actin mRNA or miR-532-3p to U6 (19).

Western blotting. Cell lysis buffer (cat. no. P0013; Beyotime Institute of Biotechnology) was used for total protein extraction of each sample and the bicinchoninic acid protein assay kit (cat. no. P0009; Beyotime Institute of Biotechnology) was used for protein concentration determination. Protein was incubated at $99^{\circ} \mathrm{C}$ for $5 \mathrm{~min}$ for denaturation. Subsequently, $40 \mu \mathrm{g}$ protein from each sample was separated via $10 \%$ SDS-PAGE, which was then transferred onto PVDF membranes. Membranes were then incubated with primary antibodies $(1: 1,000)$ against rabbit anti-NOX2 (cat. no. sc-130543; Santa Cruz Biotechnology, Inc.) anti-caspase-3 (cat. no. sc-7272; Santa Cruz Biotechnology, Inc.) and $\beta$-actin (cat. no. sc-47778; Santa Cruz Biotechnology, Inc.), followed by incubation with a horseradish peroxidase-conjugated goat anti-rabbit secondary antibody (cat. no. A0208; Beyotime Institute of Biotechnology; 1:2,000). Enhanced chemiluminescence solutions (BeyoECL Plus kit; Amersham; Cytiva) and a ChemiDox $\mathrm{XRS}^{+}$Imaging System (Bio-Rad Laboratories, Inc.) were used for protein visualization and imaging. ImageJ $1.43(\mathrm{NIH})$ was used for densitometric analysis of protein bands. Results were expressed as the ratio to $\beta$-actin.

Statistical analysis. SPSS software (version 19; IBM Corp.) was used for statistical analysis. Data are presented as the mean \pm standard deviation. One-way ANOVA followed by Tukey's post hoc test was used for statistical analysis among multiple groups. All experiments were repeated three times. $\mathrm{P}<0.05$ was considered to indicate a statistically significant difference.

\section{Results}

CI/R causes obvious brain injury in rats. TUNEL/Hoechst double staining was performed to observe cell apoptosis in brain tissues of rats subjected to I/R injury. It was found that there were significantly more TUNEL positive cells in
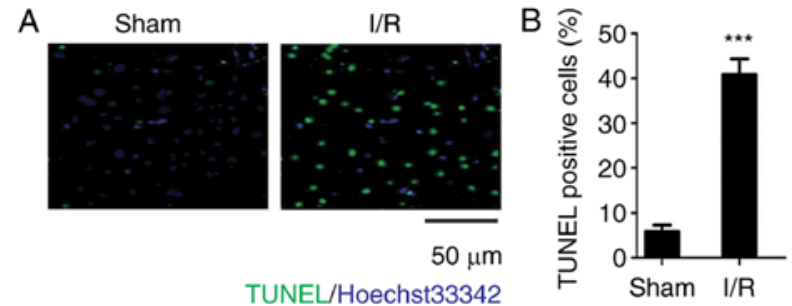

TUNEL/Hoechst33342

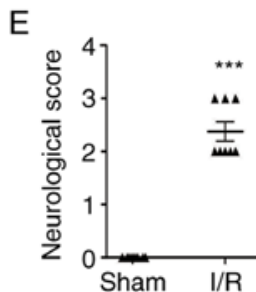

Figure 1. Cerebral I/R causes obvious brain injury of rats. (A) Representative images of TUNEL/Hoechst 33342 staining (magnification, x200). (B) TUNEL-positive cell counts (\%). (C) Infarct volume. (D) Representative images of TTC staining (coronary diameter, $2 \mathrm{~cm}$ ). (E) Neurobiological function scoring. Data are presented as the mean \pm standard deviation. I/R, rats subjected to ischemia for $2 \mathrm{~h}$ followed by reperfusion for $24 \mathrm{~h} .{ }^{* * *} \mathrm{P}<0.001 \mathrm{vs}$. sham. I/R, ischemia/reperfusion.

the $\mathrm{I} / \mathrm{R}$ brain tissues compared with the sham group, which suggested that $\mathrm{I} / \mathrm{R}$ contributes to cell apoptosis in brain tissues (Fig. 1A and B).

In accordance with the TUNEL/Hoechst double staining results, the rat brains subjected to $\mathrm{I} / \mathrm{R}$ were stained white following TTC, which indicated that the brains had undergone cerebral infarction (Fig. 1C and D). Moreover, the neurological scores of rats subjected to I/R were significantly increased compared with the shams, demonstrating reduced neurological function (Fig. 1E). Therefore, the data suggested that CI/R contributes to severe brain injury.

CI/R causes high expression of NOX2 and accumulation of $R O S$ in rat brain tissues. Since oxidative stress is one of the main causes of $\mathrm{CI} / \mathrm{R}$ injury, and NOX expression changes following CI/R injury (5), the present study focused on NOX2, a marker for ROS production. It was demonstrated that the mRNA and protein expression levels of NOX2 were significantly upregulated in the brain tissues subjected to $I / R$ injury (Fig. 2A and B), which was accompanied by a significant increase in ROS levels compared with the sham group (Fig. 2C). This suggested that NOX2 may be an important gene associated with oxidative stress in $\mathrm{CI} / \mathrm{R}$ injury.

$C I / R$ causes low expression of miR-532-3p in rat brain tissues. Subsequently, the target miRNAs of NOX2 were assessed. miR-15a, miR-16, miR-30a, miR-125b, miR-146a, miR-153, miR-223 and miR-532-3p were predicted targets of NOX 2 and it was identified that only miR-532-3p was significantly decreased (the other miRs were increased) in the brain tissues subjected to I/R injury (Fig. 3A). Moreover, the plasma expression levels of miR-532-3p were significantly decreased in rats with I/R (Fig. 3B). Thus, miR-532-3p may be a potential target of NOX2 (Fig. 3C). In addition, these data indicated that low expression of miR-532-3p may result in upregulation of NOX2. 

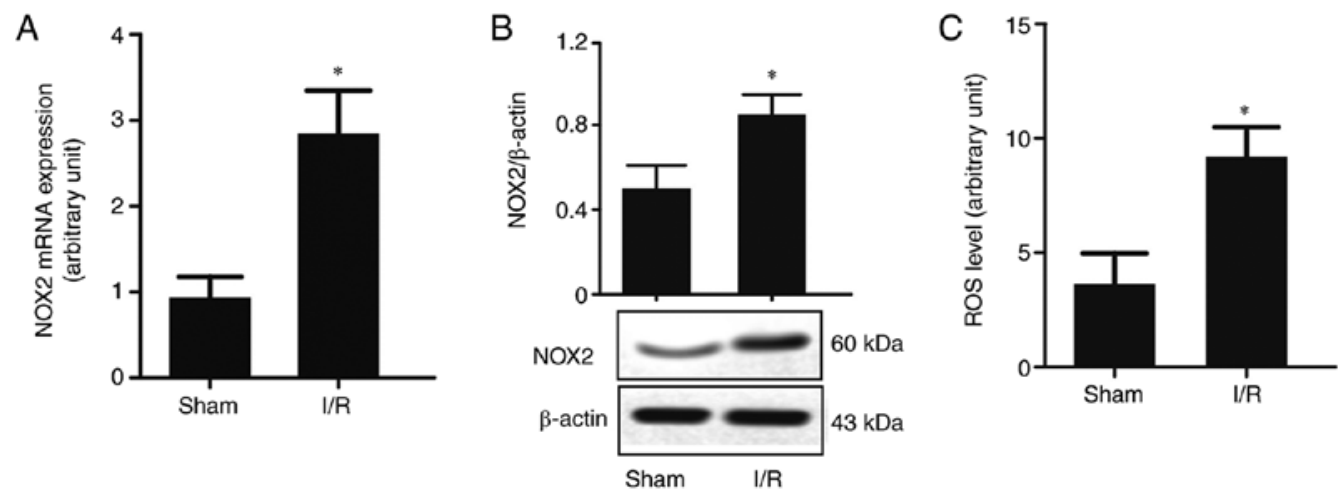

Figure 2. Cerebral I/R causes high-expression of NOX2 and accumulation of ROS in rat brain tissues. (A) NOX2 mRNA expression. (B) NOX2 protein expression. (C) ROS level. Data are presented as the mean \pm standard deviation $(\mathrm{n}=8)$. I/R, rats subjected to ischemia for $2 \mathrm{~h}$ followed by reperfusion for $24 \mathrm{~h}$. ${ }^{*} \mathrm{P}<0.05$ vs. sham. I/R, ischemia/reperfusion; NOX, NADPH oxidase; ROS, reactive oxygen species.

A
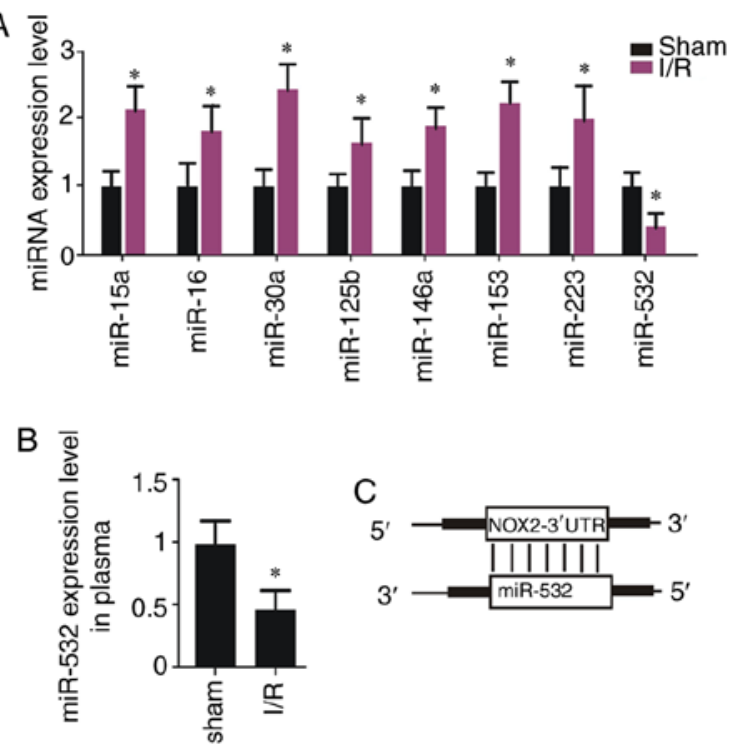

Figure 3. Cerebral I/R causes low-expression of miR-532-3p in rat brain tissues. (A) miRNA expression levels. (B) miR-532-3P expression in plasma (C) Bioinformatics analysis. Data are presented as the mean \pm standard deviation $(\mathrm{n}=8)$. I/R, rats subjected to ischemia for $2 \mathrm{~h}$ followed by reperfusion for 24 h. ${ }^{*} \mathrm{P}<0.05$ vs. sham. I/R, ischemia/reperfusion; miR, microRNA; NOX, NADPH oxidase; UTR, untranslated region.

Overexpression of miR-532-3p contributes to the expression inhibition of NOX2 in SH-SY5Y cells. miR-532-3p mimics were transfected into SH-SY5Y cells to assess its transfection efficiency, and it was identified that miR-532-3p mimics transfection significantly increased miR-532-3p expression in cells (Fig. S1), thus indicating successful transfection.

The effect of gain-of function of miR-532-3p on NOX2 expression and ROS level was examined in SH-SY5Y cells subjected to H/R injury. Compared with the control group, $\mathrm{H} / \mathrm{R}$ significantly decreased the expression of miR-532-3p in SH-SY5Y cells, while miR-532-3p mimics transfection significantly increased the expression of miR-532-3p in SH-SY5Y cells subjected to H/R (Fig. 4A). Subsequently, the expression of NOX2 was measured and it was demonstrated that the mRNA and protein expression levels of NOX2 were significantly increased in SH-SY5Y cells subjected to H/R, but miR-532-3p mimics significantly suppressed the expression of NOX2 in these cells (Fig. 4B and C). There was also a significant increase in the total NOX activity and ROS levels in SH-SY5Y cells subjected to H/R compared with control; however, miR-532-3p mimics significantly decreased these effects in the $H / R$ group (Fig. 4D-F). These results suggested that low-expression of miR-532-3p contributed, at least partly, to upregulation of NOX2.

Overexpression of $m i R-532-3 p$ decreases the apoptosis of SH-SY5Y cells. Apoptosis of SH-SY5Y cells was also measured. The expression of caspase-3 was significantly increased in cells subjected to H/R, while miR-532-3p mimic transfection significantly decreased caspase-3 expression (Fig. 5A). Furthermore, $\mathrm{H} / \mathrm{R}$ treatment significantly increased caspase-3 enzyme activity in SH-SY5Y cells, and miR-532-3p mimics intervention significantly decreased this enzyme activity (Fig. 5B).

Hoechst staining and flow cytometry results demonstrated that H/R significantly increased apoptosis and cellular death of SH-SY5Y cells; however, transfection with miR-532-3p mimics significantly reduced apoptosis and cell death caused by H/R (Fig. 5C-F). Collectively, these data indicated that miR-532-3p may protect cells from apoptosis and cell death.

miR-532-3p decreases the relative luciferase activity. A reporter gene was constructed by cloning the sequence of 3'untranslated region (UTR) of NOX2 (WT or MU) into a vector plasmid (Fig. 6A and B). Then, these plasmids containing NOX2-WT or NOX2-MU were purified and isolated by electrophoresis (Fig. 6C). The relative luciferase activity was measured and it was demonstrated that miR-532-3p mimics lead to a significant decrease in the relative luciferase activity in cells transfected with the NOX2-WT plasmid, but not with NOX2-MU (Fig. 6D). Collectively, these results suggested that miR-532-3p suppressed the expression of NOX2 by directly binding to its $3^{\prime} \mathrm{UTR}$.

\section{Discussion}

NOX-derived ROS-mediated oxidative stress serves an important role in CI/R injury (5). The aim of the present study was to identify the role of miR-532-3p in the regulation of NOX2 expression. The results indicated that miR-532-3p directly suppressed the expression of NOX2 by targeting its 3'UTR, which contributed to oxidative stress injury (Fig. 7). It was 

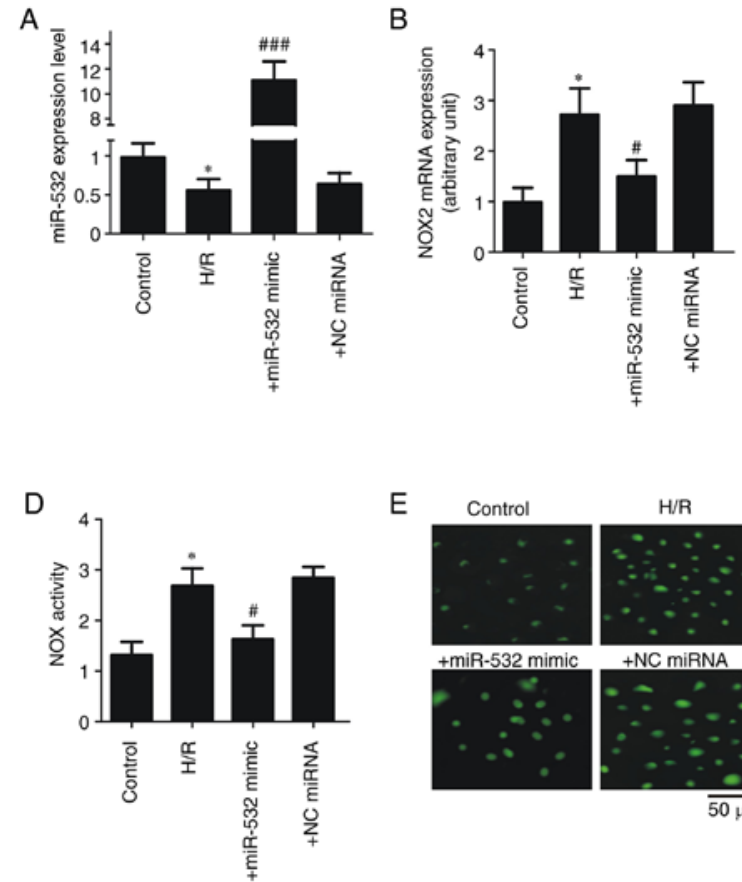

E
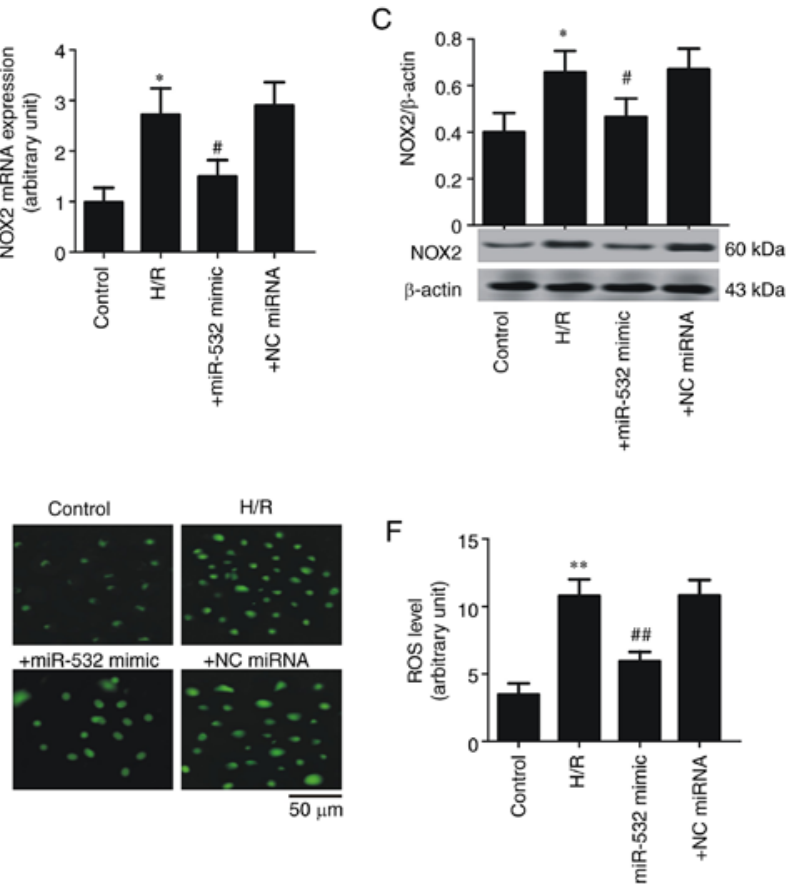

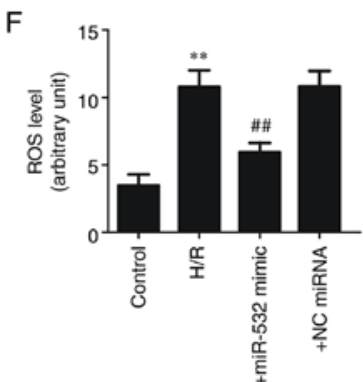

Figure 4. Overexpression of miR-532-3p contributes to the expression inhibition of NOX2 in SH-SY5Y cells. (A) miR-532-3p expression. (B) NOX2 mRNA expression. (C) NOX2 protein expression. (D) Total NOX enzyme activity. (E) Representative image of ROS level. Scale bar, $50 \mu \mathrm{m}$. (F) ROS level. Data are presented as the mean \pm standard deviation. H/R, cells subjected to $5 \mathrm{~h}$ of hypoxia followed by $20 \mathrm{~h}$ of reoxygenation; +miR-532-3p mimics, cells subjected to $\mathrm{H} / \mathrm{R}$ and treated with miR-532-3p mimic $(50 \mu \mathrm{M}) ;+\mathrm{NC}$ miRNA, cells subjected to $\mathrm{H} / \mathrm{R}$ and treated with NC miRNA $(50 \mu \mathrm{M}) .{ }^{*} \mathrm{P}<0.05$ and ${ }^{* *} \mathrm{P}<0.01 \mathrm{vs}$. control; ${ }^{*} \mathrm{P}<0.05,{ }^{\# \#} \mathrm{P}<0.01$ and ${ }^{\# \#} \mathrm{P}<0.001$ vs. H/R. H/R, hypoxia/ reoxygenation; miR/miRNA, microRNA; NOX, NADPH oxidase; NC, negative control; ROS, reactive oxygen species.
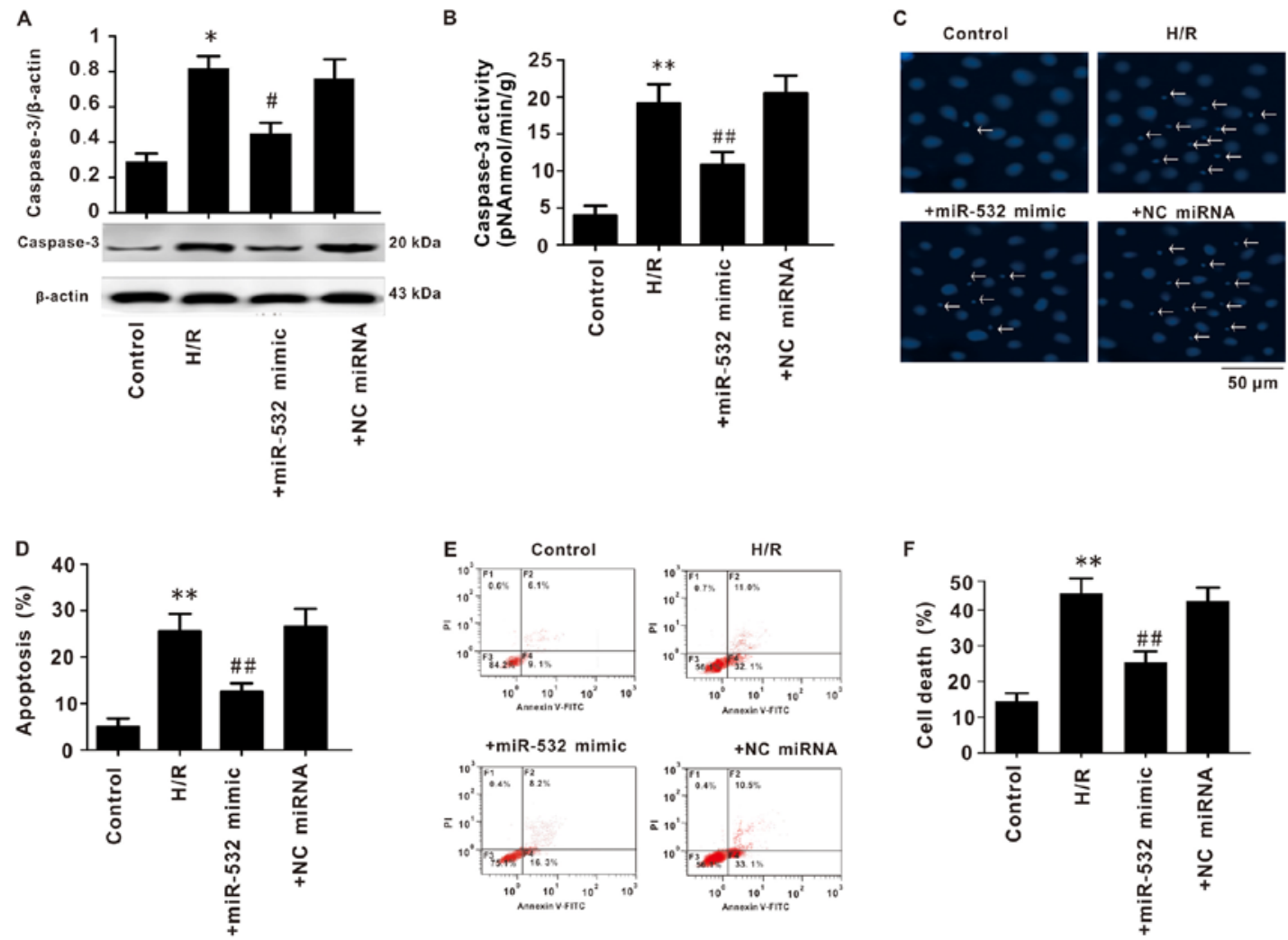

Figure 5. Overexpression of miR-532-3p decreases the apoptosis of SH-SY5Y cells. (A) Caspase-3 protein expression. (B) Caspase-3 enzyme activity. (C) Representative image of Hoechst staining (arrows indicate apoptotic bodies). Scale bar, $50 \mu \mathrm{m}$. (D) Quantification of cell apoptosis according to Hoechst staining. (E) Representative image of flow cytometry. (F) Quantification of cell death according to flow cytometry. Data are presented as the mean \pm standard deviation. $\mathrm{H} / \mathrm{R}$, cells subjected to $5 \mathrm{~h}$ of hypoxia followed by $20 \mathrm{~h}$ of reoxygenation; +miR-532-3p mimics, cells subjected to H/R and treated with miR-532-3p mimic $(50 \mu \mathrm{M}) ;+\mathrm{NC}$ miRNA, cells subjected to $\mathrm{H} / \mathrm{R}$ and treated with negative control miRNA $(50 \mu \mathrm{M})$. ${ }^{*} \mathrm{P}<0.05$ vs. control; " ${ }^{* *}<0.01$ vs. control; ${ }^{\#} \mathrm{P}<0.05$ and ${ }^{\# \prime} \mathrm{P}<0.01$ vs. H/R. H/R, hypoxia/reoxygenation; miR/miRNA, microRNA; NC, negative control; PI, propidium iodide. 


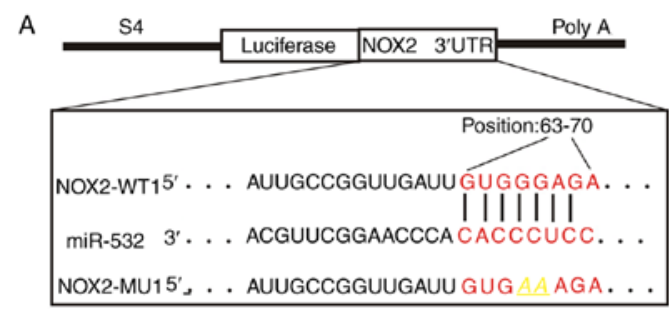

C
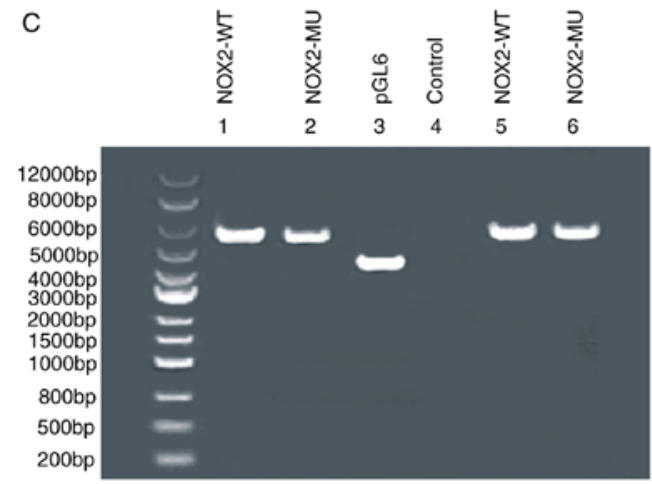

B
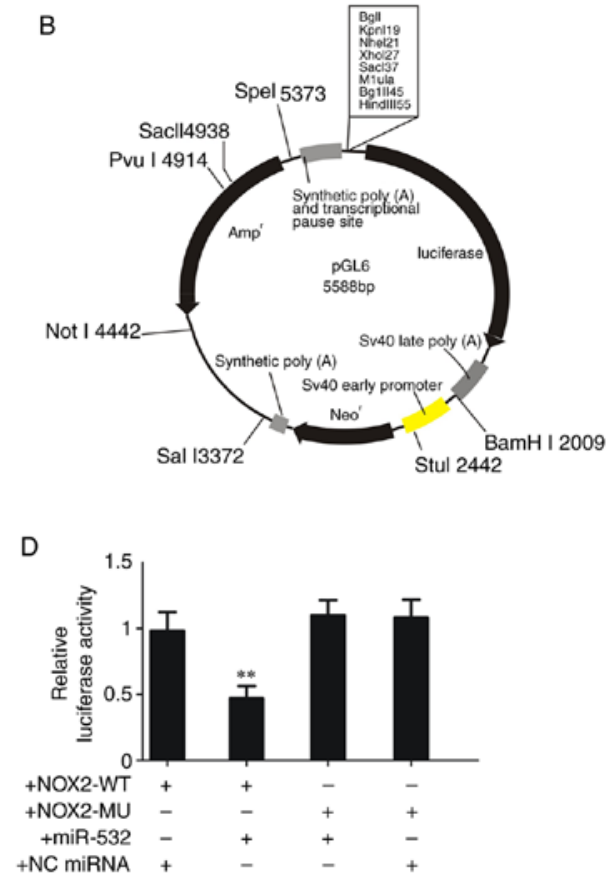

Figure 6. miR-532-3p decreases the relative luciferase activity. (A) Schematic diagram of the reporter gene plasmid containing the sequence of NOX2 targeted by miR-532-3p. (B) Schematic of the plasmid pGL6. (C) Bands of plasmid electrophoresis. (D) Relative luciferase activity. Data are presented as the mean \pm standard deviation. +NOX2-WT, cells treated with WT reporter gene plasmid NOX2-WT1 (100 ng); +NOX2-MU, cells treated with MU reporter gene plasmid NOX2-MU1 (100 ng); +miR-532-3p mimics, cells treated with miR-532-3p mimic $(50 \mu \mathrm{M})$; +NC miRNA, cells with NC miRNA (50 $\mu \mathrm{M})$. ${ }^{* *} \mathrm{P}<0.01$ vs. NOX2-WT + miR-532-3p mimics. H/R, hypoxia/reoxygenation; miR/miRNA, microRNA; NC, negative control; WT, wild-type; MU, mutant; NOX, NADPH oxidase; UTR, untranslated region.

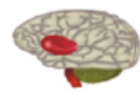

Cerebral ischemia/reperfusion injury

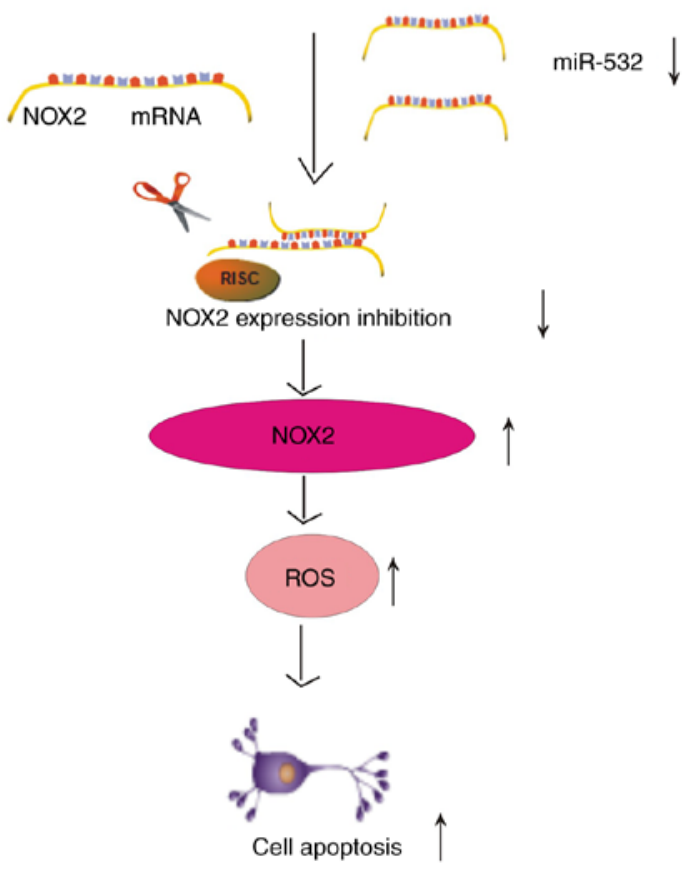

Figure 7. Schematic diagram of CI/R injury caused by miR-532-3p. Low expression of miR-532-3p contributed to a high expression of NOX2 in the brain tissues subjected to I/R injury and resulted in an increase of ROS, which contributed to neuronal apoptosis. CI/R, cerebral ischemia/reperfusion; ROS, reactive oxygen species; NOX2, NADPH oxidase 2; miR, microRNA; RISC, RNA-induced silencing complex. demonstrated that brain tissues subjected to I/R or SH-SY5Y cells exposed to $\mathrm{H} / \mathrm{R}$ had significantly increased expression levels of NOX2, as well as decreased miR-532-3p expression. Bioinformatics analysis identified that there was an interaction between miR-532-3p and NOX2. Moreover, miR-532 mimics significantly increased the expression of miR-532-3p in SH-SY5Y cells subjected to H/R, but decreased the expression of NOX2. Luciferase activity was also significantly decreased in cells co-transfected with NOX2-WT plasmid and miR-532-3p, but was not affected in cells co-transfected with the NOX2-MU plasmid. Therefore, these findings suggested that miR-532-3p may be a target for NOX2, as well as a potential biomarker and a novel target for IS.

Oxidative stress is defined as a state of ROS accumulation and an imbalance of ROS production and clearance in cells (20). ROS serve a role in the pathogenesis of numerous neurological disorders, particularly IS (21). Furthermore, ROS damage cellular macromolecules, including lipids, proteins and nucleic acids, which leads to cell injury and death (20). ROS also damage antioxidant enzymes responsible for ROS scavenging, such as glutathione peroxidase, superoxide dismutase and catalases, which results in a continuous chain reaction (5). Severe oxidative stress occurs in brain tissues subjected to ischemia for $2 \mathrm{~h}$ followed by reperfusion for $24 \mathrm{~h}$ (6). Previous studies have shown that brain tissues subjected to $\mathrm{I} / \mathrm{R}$ injury undergo an increase in ROS during the reperfusion process $(7,22)$. Reoxygenation during reperfusion provides a substrate for several enzymes in the oxidation reaction, such as xanthine oxidase, NOX and cytochromes $\mathrm{P} 450$, which produce numerous ROS, including $\mathrm{H}_{2} \mathrm{O}_{2}, \mathrm{O} 2-$ and $\mathrm{OH}(5,22)$. 
Gene expression alteration is a common mechanism for cells to respond to oxidative stress and one of the affected genes is NOX. Previous studies have reported that NOX is the main enzyme for ROS production during $\mathrm{CI} / \mathrm{R}$ injury $(5,6,22)$. Of the seven subtypes of NOX, NOX4 and particularly NOX2 are the most important for $\mathrm{CI} / \mathrm{R}$ injury $(5,22)$. It has been revealed that knockdown of NOX2 expression in rodents significantly decreases the cerebral infarction caused by MCAO (23-25). In addition, rats subjected to MCAO treated with a general or specific NOX inhibitor, such as vs2870, present with reduced brain infarction and improved neurological function (26-28). However, Kelly et al (29) reported that NOX2 inhibition with apocynin worsens stroke outcome in older rats. Possible reasons for these findings are as follows: i) ROS have dual roles (protective or harmful), if the balance between these roles becomes dysregulated, cells or tissues will be subjected to damage. For instance, NOX2 inhibition may lead to a sharp decrease in ROS level, resulting in dysregulation (5); and ii) apocynin itself exerts both therapeutic and toxic side effects, which are associated with the administration time, dosage and route. Thus, this suggests that NOX2 is a major source of ROS in brain tissues (5). In line with these previous findings, the present results indicated that $\mathrm{CI} / \mathrm{R}$ significantly increased NOX2 expression in brain tissues. Collectively, these results demonstrated that NOX2 serves a key role in $\mathrm{CI} / \mathrm{R}$ oxidative stress injury. Therefore, elucidating the regulatory mechanism of NOX2 expression is important for understanding the pathogenesis of CI/R injury, particularly for the development of novel drugs and disease therapy.

Previous studies have reported that differences in the expression patterns of miRNAs are associated with various pathophysiological processes of IS, including oxidative stress (miR-101, miR-146a, miR-410, miR-424, miR-106-5p and miR-34b), inflammation (miR-125b, miR-26a, miR-145, miR-34a, miR-9 and let-7b), excitotoxicity (miR-125b and miR-107), edema (miR-320a and miR-29a) and apoptosis (miR-125b, miR-15, miR-16, miR-34, miR-25 and miR-497) (30-38). This suggests that miRNAs have important roles in IS, particularly in the regulation of oxidative stress-related genes. NOX, especially NOX2, serves a role in the generation of ROS, which contributes to $\mathrm{CI} / \mathrm{R}$ oxidative stress injury (39). Therefore, investigating the roles of miRNAs in the regulation of NOX2 expression is of great significance for the understanding and therapy of IS. The majority of studies examining the associations of miRNAs with NOX2 have focused on cancer and cardiovascular disease (miR-34a/27b/106b/148b/2 $04 / 155 / 17 / 34 a$ ), and few studies have investigated their role in CI/R oxidative stress injury (39-43). Thus, identifying the target miRNAs of NOX2 is key to improving the understanding of $\mathrm{CI} / \mathrm{R}$ injury and facilitate the development of novel treatments. The present study identified that I/R significantly decreased miR-532-3p expression in brain tissues, as well as significantly increased the expression levels of NOX2. A previous study also demonstrated that I/R contributed to increased expression levels of NOX4 (6). However, the bioinformatics analysis indicated that miR-532-3p may be a potential target for NOX2, but not NOX4. Therefore, the present study focused on the relationship between miR-532-3p and NOX2. It was found that miR-532-3p mimics significantly decreased the expression of NOX 2 in cells subjected to $\mathrm{H} / \mathrm{R}$, as well as the relative luciferase activity. Thus, it was demonstrated that miR-532-3p may be a target of NOX2 and serves key roles in oxidative stress of I/R injury. As the roles of miRNAs in gene regulation are complex, for example, a certain gene can be targeted by different miRNAs and a specific miRNA may target different genes, the mechanisms of miRNAs targeting NOX2 remain unknown (44). In addition, whether the regulatory mechanism of miRNAs and NOX2 under normal conditions is the same as during CI/R injury requires further investigation. Furthermore, the present study only observed the effect of miR-532-3p on oxidative stress, without observing the effect of oxidative stress on miR-532-3p. Another limitation of this study is the lack of in vivo experiments to confirm the function of miR-532-3p in $\mathrm{CI} / \mathrm{R}$ injury. Therefore, further research is required prior to use of miR-532-3p for clinical therapy.

In conclusion, by directly targeting the 3'UTR of NOX2, miR-532-3p may be a regulator of NOX2 and serves important roles in $\mathrm{CI} / \mathrm{R}$ injury. To the best of our knowledge, the present study was the first to investigate the role of the miR-532-3p/NOX2 axis in CI/R oxidative stress injury, which may provide a novel target for drug development and IS treatment.

\section{Acknowledgements}

Not applicable.

\section{Funding}

This work was funded by the National Natural Science Foundation of China (grant no. 81603107), the Education Department of HuNan Province (grant no. 18C1853) and ChangSha Science \& Technology Bureau (grant no. kq1801125).

\section{Availability of data and materials}

The datasets used and /or analyzed during the current study are available from the corresponding author on reasonable request.

\section{Authors' contributions}

LM, MLZ, APW, YT and LCD performed the experiments. TML and DBK contributed to the data analysis and manuscript drafting. GLS and ZBY contributed to the design of the experiments and drafting of the manuscript. All authors have read and approved the manuscript.

\section{Ethics approval and consent to participate}

Animal experiments were conducted following the Guide for the Care and Use of Laboratory Animals, and were approved by the Animal Ethics Committee of Hunan Normal University.

\section{Patient consent for publication}

Not applicable.

\section{Competing interests}

The authors declare that they have no competing interests. 


\section{References}

1. Randolph SA: Ischemic Stroke. Workplace Health Saf 64: 444 2016.

2. Khoshnam SE, Winlow W, Farzaneh M, Farbood Y and Moghaddam HF: Pathogenic mechanisms following ischemic stroke. Neurol Sci 38: 1167-1186, 2017.

3. Enzmann G, Kargaran S and Engelhardt B: Ischemia-reperfusion injury in stroke: Impact of the brain barriers and brain immune privilege on neutrophil function. Ther Adv Neurol Disord 11: 1756286418794184,2018

4. Siti HN, Kamisah Y and Kamsiah J: The role of oxidative stress, antioxidants and vascular inflammation in cardiovascular disease (a review). Vascul Pharmacol 71: 40-56, 2015.

5. Ma MW, Wang J, Zhang Q, Wang R, Dhandapani KM, Vadlamudi RK and Brann DW: NADPH oxidase in brain injury and neurodegenerative disorders. Mol Neurodegener 12: 7, 2017.

6. Lou Z, Wang AP, Duan XM, Hu GH, Song GL, Zuo ML and Yang ZB: Upregulation of NOX2 and NOX4 mediated by TGF- $\beta$ signaling pathway exacerbates cerebral ischemia/reperfusion oxidative stress injury. Cell Physiol Biochem 46: 2103-2113, 2015.

7. Sun JB, Li Y, Cai YF, Huang Y, Liu S, Yeung PK, Deng MZ, Sun GS, Zilundu PL, Hu QS, et al: Scutellarin protects oxygen/glucose-deprived astrocytes and reduces focal cerebral ischemic injury. Neural Regen Res 13: 1396-1407, 2015.

8. Xu W, Gao L, Zheng J, Li T, Shao A, Reis C, Chen S and Zhang J: The roles of microRNAs in stroke: Possible therapeutic targets. Cell Transplant 27: 1778-1788, 2018.

9. Majdi A, Mahmoudi J, Sadigh-Eteghad S, Farhoudi M and Shotorbani SS: The interplay of microRNAs and post-ischemic glutamate excitotoxicity: An emergent research field in stroke medicine. Neurol Sci 37: 1765-1771, 2016.

10. Mao L, Zuo ML, Hu GH, Duan XM and Yang ZB: mir-193 targets ALDH2 and contributes to toxic aldehyde accumulation and tyrosine hydroxylase dysfunction in cerebral ischemia/reperfusion injury. Oncotarget 8: 99681-99692, 2017.

11. Yang ZB, Zhang Z, Li TB, Lou Z, Li SY, Yang H, Yang J, Luo XJ and Peng J: Up-regulation of brain-enriched miR-107 promotes excitatory neurotoxicity through down-regulation of glutamate transporter-1 expression following ischaemic stroke. Clin Sci 127: 679-689, 2014

12. Liu Z, Tuo YH, Chen JW, Wang QY, Li S, Li MC, Dai G, Wang JS, Zhang YL, Feng L and Shi ZS: NADPH oxidase inhibitor regulates microRNAs with improved outcome after mechanical reperfusion. J Neurointerv Surg 9: 702-706, 2017.

13. Ma J, Zhang J, Wang Y, Long K, Wang X, Jin L, Tang Q, Zhu L, Tang G, Li X and Li M: MiR-532-5p alleviates hypoxia-induced cardiomyocyte apoptosis by targeting PDCD4. Gene 675: 36-43, 2018.

14. Sun W, Liu Q, Leng J, Zheng Y and Li J: The role of Pyruvate Dehydrogenase Complex in cardiovascular diseases. Life Sci 121: 97-103, 2015.

15. Li P, Teng F, Gao F, Zhang M, Wu J and Zhang C: Identification of circulating microRNAs as potential biomarkers for detecting acute ischemic stroke. Cell Mol Neurobiol 35: 433-447, 2015.

16. Sepramaniam S, Tan JR, Tan KS, DeSilva DA, Tavintharan S, Woon FP, Wang CW, Yong FL, Karolina DS, Kaur P, et al: Circulating microRNAs as biomarkers of acute stroke. Int J Mol Sci 15: 1418-1432, 2014

17. National Research Council (US) Committee for the Update of the Guide for the Care and Use of Laboratory Animals: Guide for the Care and Use of Laboratory Animals, 8th edition. National Academicals Press (US), Washington, DC, 2011.

18. Whiteside G, Cougnon N, Hunt SP and Munglani R: An improved method for detection of apoptosis in tissue sections and cell culture, using the TUNEL technique combined with Hoechst stain. Brain Res Protoc 2: 160-164, 1998

19. Livak KJ and Schmittgen TD: Analysis of relative gene expression data using real-time quantitative PCR and the 2(-Delta Delta C(T)) method. Methods 25: 402-408, 2001.

20. Brandes RP, Weissmann N and Schröder K: Redox-mediated signal transduction by cardiovascular Nox NADPH oxidases. J Mol Cell Cardio 73: 70-79, 2014.

21. Patel M: Targeting oxidative stress in central nervous system disorders. Trends Pharmacol Sci 37: 768-778, 2016.

22. Awooda HA, Lutfi MF, Sharara GGM and Saeed AM Oxidative/nitrosative stress in rats subjected to focal cerebral ischemia/reperfusion. Int J Health Sci (Qassim) 9: 17-24, 2015.
23. Davis SM and Pennypacker KR: Targeting antioxidant enzyme expression as a therapeutic strategy for ischemic stroke. Neurochem Int 107: 23-32, 2017.

24. De Silva TM, Brait VH, Drummond GR, Sobey CG and Miller AA: Nox2 oxidase activity accounts for the oxidative stress and vasomotor dysfunction in mouse cerebral arteries following ischemic stroke. PLoS One 6: e28393, 2011.

25. Wang Z, Wei X, Liu K, Zhang X, Yang F, Zhang H, He Y, Zhu T, Li F, Shi W, et al: NOX2 deficiency ameliorates cerebral injury through reduction of complexin II-mediated glutamate excitotoxicity in experimental stroke. Free Radic Biol Med 65: 942-951, 2013.

26. Jackman KA, Miller AA, De Silva TM, Crack PJ, Drummond GR and Sobey CG: Reduction of cerebral infarct volume by apocynin requires pretreatment and is absent in Nox2-deficient mice. Br J Pharmacol 156: 680-688, 2009.

27. Wingler K, Altenhoefer SA, Kleikers PWM, Radermacher KA, Kleinschnitz C and Schmidt HH: VAS2870 is a pan-NADPH oxidase inhibitor. Cell Mol Life Sci 69: 3159-3160, 2012.

28. Surace MJ and Block ML: Targeting microglia-mediated neurotoxicity: The potential of NOX2 inhibitors. Cell Mol Life Sci 69: 2409-2427, 2012.

29. Kelly KA, Li X, Tan Z, VanGilder RL, Rosen CL and Huber JD: NOX2 inhibition with apocynin worsens stroke outcome in aged rats. Brain Res 1292, 165-172, 2009.

30. Liu NN, Dong ZL and Han LL: MicroRNA-410 inhibition of the TIMP2-dependent MAPK pathway confers neuroprotection against oxidative stress-induced apoptosis after ischemic stroke in mice. Brain Res Bull 143: 45-57, 2018.

31. Wang SW, Liu Z and Shi ZS: Non-Coding RNA in acute ischemic stroke: Mechanisms, biomarkers and therapeutic targets. Cell Transplant 27: 1763-1777, 2018.

32. Huang R, Ma J, Niu B, Li J, Chang J, Zhang Y, Liu P and Luan X: MiR-34b protects against focal cerebral ischemia-reperfusion (I/R) injury in rat by targeting keap1. J Stroke Cerebrovasc Dis 28: 1-9, 2019.

33. Zheng Y, Pan C, Chen M, Pei A, Xie L and Zhu S: miR-29a ameliorates ischemic injury of astrocytes in vitro by targeting the water channel protein aquaporin 4. Oncol Rep 41: 1707-1717, 2019.

34. Deng Y, Ma G, Dong Q, Sun X, Liu L, Miao Z and Gao F: Overexpression of miR-224-3p alleviates apoptosis from cerebral ischemia reperfusion injury by targeting FIP200. J Cell Biochem 1200: 17151-17158, 2019.

35. Fu C, Chen S, Cai N, Liu Z, Wang P and Zhao J: Potential neuroprotective effect of miR-451 against cerebral ischemia/reperfusion injury in stroke patients and a mouse model. World Neurosurgery 130: e54-e61, 2019.

36. Rink $\mathrm{C}$ and Khanna S: MicroRNA in ischemic stroke etiology and pathology. Physiol Genomics 43: 521-528, 2011.

37. Tan JR, Koo YX, Kaur P, Liu F, Armugam A, Wong PT and Jeyaseelan K: microRNAs in stroke pathogenesis. Curr Mol Med 11: 76-92, 2011

38. Zhao H, Han Z, Ji X and Luo Y: Epigenetic regulation of oxidative stress in ischemic stroke. Aging Dis 7: 295-306, 2016.

39. Kahles T and Brandes RP: Which NADPH oxidase isoform is relevant for ischemic stroke? The case for nox 2. Antioxid Redox Signal 18: 1400-1417, 2013.

40. Li J, Hui L, Kang Q and Li R: Down-regulation of microRNA-27b promotes retinal pigment epithelial cell proliferation and migration by targeting Nox2. Pathol Res Pract 214: 925-933, 2018.

41. Kim SM, Hur DY, Hong SW and Kim JH: EBV-encoded EBNA1 regulates cell viability by modulating miR34a-NOX2-ROS signaling in gastric cancer cells. Biochem Biophys Res Commun 494: 550-555, 2017.

42. Yang J, Brown ME, Zhang H, Martinez M, Zhao Z, Bhutani S, Yin S, Trac D, Xi JJ and Davis ME: High-throughput screening identifies microRNAs that target Nox2 and improve function after acute myocardial infarction. Am J Physiol Heart Circ Physio 312: H1002-H1012, 2017.

43. Wang XH and Wang TL: MicroRNAs of microglia: Wrestling with central nervous system disease. Neural Regen Res 13: 2067-2072, 2018.

44. Ambros V: The functions of animal microRNAs. Nature 431: 350-355, 2004.

This work is licensed under a Creative Commons Attribution-NonCommercial-NoDerivatives 4.0 International (CC BY-NC-ND 4.0) License. 